\title{
Moyamoya disease: functional and neurocognitive outcomes in the pediatric and adult populations
}

\author{
David G. Weinberg, B.S., Rudy J. Rahme, M.D., Salah G. Aoun, M.D., \\ H. Hunt Batjer, M.D., AND Bernard R. BendoK, M.D. \\ Department of Neurological Surgery, Northwestern University Feinberg School of Medicine; and McGaw \\ Medical Center, Chicago, Illinois
}

\begin{abstract}
Object. Moyamoya disease is an occlusive cerebrovascular disorder commonly resulting in neurocognitive impairment. The cognitive outcome parameters commonly affected are intelligence, memory, executive function, and quality of life. In this paper, the authors review the existing literature on cognitive and clinical outcomes in adult and pediatric moyamoya populations separately.

Methods. A systematic review of the cognitive and clinical outcome literature was performed using the PubMed/ MEDLINE database. Outcomes data were contrasted between adult and pediatric populations.

Results. Intelligence is the main cognitive outcome parameter affected in pediatric patients with moyamoya disease, whereas adults most commonly suffer from executive function impairment. Memory has not been studied sufficiently in pediatric patients, and its dysfunction in the adult population remains controversial. Quality of life has not been studied appropriately in either population. Surgical revascularization is the only beneficial treatment option, and a combination of direct and indirect bypass techniques has shown benefit, but the impact on the above-mentioned parameters has not been sufficiently elucidated.

Conclusions. Moyamoya disease affects the cognition and daily function in pediatric patients to a greater extent than in adult patients. Due to the rarity of the disease, there is a distinct lack of high-level evidence regarding cognitive and clinical outcomes. (DOI: 10.3171/2011.3.FOCUS1150)
\end{abstract}

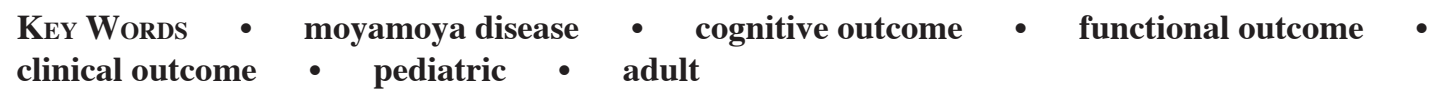

$\mathrm{M}$ OYAMOYa disease is a chronic cerebrovascular condition characterized by progressive stenosis of the arteries of the circle of Willis. This progressive occlusion leads to the recruitment of a large number of collateral vessels, giving moyamoya its characteristic "puff of smoke" appearance on angiographic studies..$^{2,14,26,29}$ The Ministry of Health and Welfare of Japan has subdivided moyamoya presentation into 4 categories: ischemic (63.4\%), hemorrhagic (21.6\%), epileptic $(7.6 \%)$, and "other" $(7.5 \%) .{ }^{11}$ However, these categories are not mutually exclusive; patients with moyamoya disease can have hemorrhagic transformation of an ischemic stroke. ${ }^{29}$

Moyamoya disease is most prevalent in Asian popu-

Abbreviations used in this paper: $\mathrm{CBF}=$ cerebral blood flow; CVRS-II = California Visual Reproduction Subtest-Second Edition; DCS = dysexecutive cognitive syndrome; D-KEFS = Delis-Kaplan Executive Function System; EDAS = encephaloduroarteriosynangiosis; FSIQ = full-scale IQ; $\mathrm{mRS}=$ modified Rankin Scale; OEF = oxygen extraction fraction; PIQ = performance IQ; QOL = quality of life; $\mathrm{rCBV}=$ regional cerebral blood volume; $\mathrm{rTTP}=$ regional time to peak; SF-36 = 36-Item Short Form Health Survey; TIA = transient ischemic attack; VIQ = verbal IQ; WMT-RVRS = Wechsler Memory Test-Revised Visual Reproduction Subtest. lations and demonstrates unique epidemiological features in these populations compared with North American patients. ${ }^{47}$ In the Asian population, moyamoya disease has a bimodal age of onset, with one peak in the 1st decade and another peak in the 3rd and 4th decades. ${ }^{26}$ In addition, the disease process in children mainly causes ischemic symptoms due to inadequate perfusion, whereas in adults hemorrhage related to the fragility of the neovessels is more common. ${ }^{2,29,47}$ In contrast, in the US, the most common mode of presentation is ischemia in both children and adults, whereas a hemorrhagic presentation is relatively rare $(12 \%-17 \%){ }^{4,47}$ Furthermore, the bimodal age distribution is not as clear in studies based on patients of North American descent. ${ }^{2,4}$ It is important to note, though, the racial diversity of North American cohorts with patients of various ethnic backgrounds as compared with Asian cohorts composed primarily of patients of Asian descent ${ }^{49}$ It has been observed that an ischemic presentation in North American patients may possibly carry a more benign course of the disease. ${ }^{4}$ However, this has not yet been validated, and conclusive evidence that cognitive and clinical outcomes differ between Asian and North American patients is not yet present in the literature.

In patients with moyamoya disease, cognitive and 
intellectual functioning is often affected. ${ }^{21,30,36}$ The majority of outcome literature has focused on the neuropsychological aspect of children with the disease, whereas few studies have analyzed this outcome in adults. Vessel stenosis has been shown to progress at a faster rate in pediatric patients than in adults.,14,26 This progression has been associated with lower intelligence levels in pediatric patients. ${ }^{26}$ The parameter that has been most commonly used to assess the mental status of moyamoya patients is the FSIQ. Other parameters include memory, executive functioning, and QOL. Clinical outcome has also been studied, with a specific focus on recurrent TIAs, cerebral infarction, and hemorrhage.

Moyamoya treatment aims at improving cerebral perfusion and decreasing neovessel formation, thus preventing future hemorrhagic and/or ischemic events. ${ }^{14}$ No medical treatment has been proven efficacious. Surgical treatment can be broken down into 3 categories: direct, indirect, and combined revascularization. The direct bypass procedure involves the anastomosis of a branch of the external carotid artery, typically the superficial temporal artery with the middle cerebral artery., ${ }^{2,13}$ Indirect revascularization involves the transposition of a biological vascularized graft onto the ischemic region of the brain, therefore inducing growth of new vessels. Various indirect techniques exist. Encephalomyosynangiosis involves use of the temporalis muscle, and is one of the most commonly applied techniques. ${ }^{33}$ Another common indirect procedure is EDAS, in which an external carotid artery branch, typically the superficial temporal artery, is transposed to the ischemic tissue..$^{47}$ The dural layer may also be folded into the subdural space to induce more neovessel formation. ${ }^{28}$ The combination of these techniques, encephaloduroarteriomyosynangiosis, is used to provide further revascularization. ${ }^{22}$ Grafts that are used less commonly include the pericranial flap, omental tissue, and gracilis muscle. ${ }^{31,41}$

\section{Methods}

For this review, a systematic search of the Englishlanguage literature was performed with the PubMed/ MEDLINE database by searching for the following keywords and various combinations thereof: "Moyamoya Disease," "Surgery," "Treatment," and "Outcome." This search initially retrieved 29 articles, which were reviewed and separated into pediatric and adult categories. The reference lists of the relevant articles were reviewed for additional sources, and 55 articles were included in the final review.

\section{Pediatric Outcomes}

\section{Cognitive Outcomes}

Intelligence. Intelligence is the most studied cognitive parameter in pediatric patients with moyamoya disease. The most commonly used intelligence tests are the Wechsler Intelligence Scale and its variations, including the Wechsler Intelligence Scale for Children-Revised (WISC-R), the Wechsler Preschool and Primary Scale of Intelligence (WPPSI), and the Wechsler Intelligence
Scale for Children-Third Edition (WISC-III). ${ }^{21,30,36}$ The FSIQ has 2 components: the VIQ and the PIQ. The VIQ is a measure of verbal comprehension and working memory, whereas the PIQ is a measure of perceptual organization and processing speed. ${ }^{36}$ The extent of cognitive impairment has been correlated with the degree of vascular occlusion. Hence, the effect of revascularization surgery, direct and/or indirect, on cognitive functioning has been the focus of multiple studies. . $^{32,34,35}$

In patients with moyamoya disease, intelligence is commonly diminished relative to the general population. ${ }^{19,30,32}$ In one study, Kuroda et al.$^{30}$ measured the FSIQ scores of 52 pediatric Japanese patients and compared these with scores from the general Japanese population. A significantly higher proportion of moyamoya patients were mentally impaired (as defined by an FSIQ score < 70) than were controls (15.4\% and $2.2 \%$, respectively). There were no significant differences between moyamoya patients and controls in all other intelligence score ranges.

One point of controversy is whether the diminished intelligence is due to a secondary condition accompanying moyamoya disease or is directly a product of the disease process. Hogan et al. ${ }^{16}$ provided evidence for the latter in their analysis of intellectual function in non-Japanese children with moyamoya who also had sickle cell anemia. The prevalence of sickle cell anemia in moyamoya patients is higher than in the general population. Participants were grouped into patients with both moyamoya disease and sickle cell anemia, and a control group of patients with sickle cell anemia alone. Intelligence scores in children in the group with moyamoya disease and sickle cell anemia were significantly lower than those in the control group (VIQ, $\mathrm{p}=0.003$; PIQ, $\mathrm{p}=0.002$ ). ${ }^{16}$ Additionally, a statistically significant reduction in PIQ independent of sickle cell anemia was seen in patients with moyamoya disease relative to those without moyamoya disease $(\mathrm{p}=0.004)$, indicating a probable direct link between the moyamoya disease process and intelligence regression. ${ }^{16}$

Moyamoya disease has been postulated to cause progressive cognitive decline. Serial intelligence testing has been used to analyze this deterioration throughout the course of the disease..$^{1,19,24}$ Suzuki and Takaku ${ }^{48}$ defined angiographic stages in relation to the degree of occlusion. The stenoses have been shown to progress until adolescence and stabilize by the age of 20 years. ${ }^{8}$ Furthermore, older children tend to have worse cognitive impairment than younger children. ${ }^{1,17,19,24}$ Imaizumi et al..$^{19}$ stratified patients by disease period and measured serial IQ scores. Patients with the disease for $<5$ years showed significantly higher IQs than those afflicted for 5-10 years or for > 10 years $(p=0.004$ and $p=0.001$, respectively). However, no significant difference in intelligence existed between patients whose disease had been diagnosed $>5$ years earlier and those who received their diagnosis $>10$ years earlier. These results mimic the trend of disease progression. In another study, Ishii et al. ${ }^{21}$ stratified preoperative patients by age and found that the mean FSIQ of older pediatric patients was lower than that of younger pediatric patients (patients between 13 and 16 years old: FSIQ $=82.6 \pm 16.4$; patients between 5 and 8 years old: FSIQ $=$ $108.0 \pm 12.9$ [mean $\pm \mathrm{SD}])$. 


\section{Neurocognitive outcomes in patients with moyamoya disease}

Surgical revascularization has been proven beneficial in slowing cognitive decline in pediatric moyamoya patients. ${ }^{20,32,34,35,50}$ The cognitive effects of EDAS were studied by Matsushima et al. ${ }^{34}$ in 65 pediatric moyamoya patients. The authors measured intelligence by using IQ scores for children $>5$ years of age and development quotient scores for children $<5$ years. Although there was no improvement postoperatively, the surgery halted the cognitive decline, with intelligence scores stabilizing postoperatively. In another study, the same group of authors found that patients had normal intelligence levels 9.5 years postoperatively, given a preoperative FSIQ of $>$ $70(\mathrm{FSIQ}=100.0 \pm 15.5 ; \mathrm{VIQ}=100.0 \pm 15.7 ; \mathrm{PIQ}=100.2$ \pm 16.3). ${ }^{36}$ However, when disease onset occurs before the 3rd year of life, cognitive function has been shown not to improve. ${ }^{35}$

Decreased CBF has been correlated with diminished intelligence. ${ }^{44}$ Ishii et al. ${ }^{21}$ correlated pre- and postoperative CBF levels with IQ scores in moyamoya patients. Preoperatively, patients with lower FSIQ scores generally had lower CBF values. Postoperatively, FSIQ improved or remained unchanged in 14, and PIQ improved or remained unchanged in 13 of the 15 tested patients. The patients with improved intelligence showed corresponding $\mathrm{CBF}$ improvement.

Although intelligence scores generally stabilize after surgical revascularization, this is not always the case. $^{21,30,34,35,50}$ Kuroda et al. ${ }^{30}$ attributed poor cognitive outcome to 2 independent factors: 1) completed stroke as opposed to TIA (OR 33.4, 95\% CI 2.4-474), and 2) "small craniotomy" surgery (OR 19.6, 95\% CI 1.8-215). Revascularization by indirect bypass can only occur within the surgical window, because vascularized grafts can only be as large as this window. Furthermore, frontal lobe $\mathrm{CBF}$ has been correlated with intellectual development. ${ }^{10}$ Because "small craniotomy" moyamoya surgeries are generally confined to the temporoparietal region, intelligence may not fully recover. Therefore, Kuroda et al. concluded that a "large craniotomy" is necessary to provide maximal revascularization and therefore obtain the best cognitive outcome. ${ }^{23,44}$ This issue needs further study and must be measured against the risk incurred to collateral dural-to-pial anastomoses, which could be threatened by a larger craniotomy.

Memory. The vast majority of studies regarding cognitive outcome of pediatric moyamoya patients have focused on intelligence as a measure of higher functioning. Although there have not been many studies concerning memory, the present literature implies that memory is not impaired relative to other cognitive parameters. One study reported the neuropsychological testing of 2 pediatric moyamoya patients, in which memory was one of the parameters analyzed. ${ }^{1}$ The authors used sections of the Wide Range Assessment of Memory and Learning test, including picture memory, story memory, design memory, and sentence memory to assess memory pre- and postoperatively. Although these measures were generally not impaired preoperatively, they still showed a notable gain in function postoperatively.

Executive Function. Executive function is the abil- ity to plan and perform a series of actions in an effort to accomplish a specific motor goal. The integrity of the dorsolateral prefrontal cortices of the frontal lobes is required for this function. ${ }^{10}$ This function has not been examined in pediatric moyamoya patients.

Quality of Life. There are very few papers exploring the QOL of children with moyamoya disease. The potential improvement in QOL postoperatively is a major consideration in the decision for surgical intervention. Guzman et al. ${ }^{14}$ prospectively reported on the QOL in both adult and pediatric moyamoya patients treated at Stanford University Medical Center. The series involved 450 revascularization procedures performed in 329 patients, 96 of whom were children. The mRS was used as a QOL assessment tool. The authors found that $71.2 \%$ of patients had significantly improved postoperative mRS scores. Improvement by $1-2$ points on the $\mathrm{mRS}$ was seen in $67.8 \%$ of patients. The best predictor of a good postoperative mRS score (0-2) was a preoperative mRS score of $\leq 2$ (OR 26, $\mathrm{p}<0.0001)$. Whereas the mRS score is a measure of disability, QOL is a much broader parameter that takes into account multiple dimensions, including physical, psychological, and social health. Quality of life measures such as the SF-36 have been used successfully to examine long-term QOL in stroke patients. ${ }^{42}$ A multiple-domain test such as the SF36 would give a more complete understanding of QOL in moyamoya patients, as it has in stroke patients. However, as a first measure, Guzman et al. provided an interesting insight into the postoperative improvement in QOL of moyamoya patients.

\section{Clinical Outcome}

Clinical outcome studies have focused on TIA, cerebral infarction, and hemorrhage. In one study of conservatively treated pediatric patients, TIA occurred most frequently during the first 4 years after disease onset, with the incidence declining thereafter. ${ }^{32}$ Surgical revascularization has been shown to decrease the incidence of stroke and TIA. ${ }^{13,22,25,38,45}$ Matsushima et al. ${ }^{34}$ quantified the regression in TIA incidence by studying 65 pediatric moyamoya patients pre- and post-EDAS. Patients continued to have ischemic events until a mean of 239 days postoperatively, after which no ischemic events were recorded. Symptoms including headache, seizure, and involuntary movements disappeared after the last ischemic event. The authors concluded that EDAS effectively eliminates long-term ischemic episodes.

The major predictors of clinical outcome in the pediatric population remain controversial. Darwish and Besser ${ }^{5}$ hypothesized that postoperative clinical outcome primarily correlated with clinical status at presentation. These authors studied 16 children with moyamoya disease and examined trends in mRS scores pre- and postoperatively. These mRS scores and the number of postoperative ischemic events only correlated with the clinical presentation and were not related to the type of revascularization procedure or patient age.

Other studies have analyzed predictors of clinical outcome from the perspective of hemodynamic parameters. Kim et al. ${ }^{28}$ retrospectively analyzed 67 pediatric moya- 
moya patients, and found the main predictors of long-term clinical outcome to be age at onset, type of surgical revascularization procedure, and postoperative cerebral hemodynamics. Furthermore, after analyzing 77 pediatric moyamoya patients by using postoperative basal/acetazolamide stress brain perfusion SPECT at 6 and 12 months postoperatively, So et al. ${ }^{46}$ found that clinical outcome correlated best with cerebrovascular reserve. In addition, these authors measured a cerebrovascular reserve index and quantitatively showed a correlation between vascular reserve and clinical outcome. They concluded that postoperative SPECT results and cerebrovascular reserve index values predict clinical outcome in pediatric moyamoya patients.

Cerebral blood volume might also be used as a predictor of clinical outcome. ${ }^{53}$ Quantitative MR perfusion imaging can be used to study hemodynamic parameters, including rTTP and rCBV. Using this imaging technique, Yun et al. ${ }^{53}$ found that the rTTP was significantly shorter postoperatively than preoperatively $(\mathrm{p}<0.001)$ and that the rCBV was significantly lower postoperatively than preoperatively $(\mathrm{p}=0.014)$. Large rTTP delays and high rCBV levels preoperatively are due to dispersion of contrast material throughout the extensive network of collateral vessels. These values are both decreased postoperatively. Also, change in the rTTP was a significant predictor of clinical outcome, whereas change in the rCBV showed no such correlation.

\section{Adult Outcomes}

\section{Cognitive Outcomes}

Intelligence. In the few reports regarding intelligence in adult moyamoya patients, cognition has been shown to be less affected in adults than in children..$^{9,14,26}$ Karzmark et al. ${ }^{26}$ confirmed this finding by measuring IQ scores with the Wechsler Adult Intelligence Scale-Third Edition (WAIS-III) in 36 adult patients. The mean FSIQ score was 95. The normal intelligence levels seen in adult patients contrast with the generally diminished intelligence seen in the pediatric moyamoya population. Further evidence was provided with the neuropsychological testing of 29 adults in which various forms of the adult Wechsler scales for intelligence were used. ${ }^{9}$ The adult patients had a mean IQ score of $98.7 \pm 17.2$, which is comparable to that of the general population.

Memory. Memory has been studied more extensively in adult patients than in children. However, there is still controversy regarding the effects of moyamoya disease on memory in the adult population. The 2 main tests that have been used to examine adult memory function are the CVRS-II and the WMT-RVRS. Festa et al. ${ }^{9}$ used neuropsychological testing to assess memory function in 29 adult patients. At initial presentation, $31 \%$ of patients showed verbal memory dysfunction on the delayed list recall test. The overall memory domain score was 1.1 SDs below the mean. The memory domain score is defined as the mean of $\mathrm{z}$ scores for immediate recall, delayed recall, and list recognition tests. The authors also studied working memory, which is a reflection of attention span, with $21 \%$ of patients falling lower than 1.5 SDs below the mean. The authors localized the impaired cognitive functions, including memory, to subcortical and frontal systems. The fact that these regions do not correspond with the regions of ischemia implies that the neurocognitive impairment may also be due to chronic hypoperfusion, in addition to acute localized ischemic events. Based on this finding, the authors concluded that overall cognitive dysfunction might be an indication for surgical intervention. ${ }^{9}$

Other studies have found memory to be unaffected in adult patients. Using subsets of the CVRS-II and WMT-RVRS, Karzmark et al. ${ }^{26}$ analyzed memory in 36 adult moyamoya patients. Of all cognitive parameters tested, memory showed the lowest rates of impairment. Therefore, the authors concluded that medial temporal function is relatively spared in patients with moyamoya disease.

Executive Function. Executive function has also been studied more extensively in adults than in children. The major dimensions of executive function include flexibility of thinking, problem solving, motor planning, impulse control, and concept formation. The main tests that have been used to measure these dimensions are the D-KEFS Design Fluency Test, the Letter and Category Fluency Tests, and the Trail-Making Test Part B. The D-KEFS test assesses flexibility of thinking, impulse control, and planning. ${ }^{6}$ The Letter and Category Fluency Tests examine semantic knowledge. ${ }^{12}$ The Trail-Making Test Part B analyzes frontal lobe function in terms of problem solving and motor planning. ${ }^{54}$

Analysis of existing literature revealed executive function to be considerably impaired in adult moyamoya patients. ${ }^{9,26}$ Festa et al. ${ }^{9}$ found the executive function of 29 adult patients to be significantly diminished, with a mean Trail-Making Test Part B score that was 1.3 SDs below the mean. Karzmark et al. ${ }^{26}$ found executive function to be the most impaired cognitive parameter in moyamoya patients. Of 36 adult patients, $11 \%$ showed impairment on the D-KEFS test, and $42 \%$ showed impairment on the Trail-Making Test Part B. Furthermore, $47 \%$ of patients showed Letter Fluency impairment and 44\% showed Category Fluency impairment. Due to its role in executive function, the frontal lobe is thought to be directly affected in adult moyamoya patients.

Quality of Life. An extensive look at QOL in this population has not yet been performed. Some elements of QOL, including $\mathrm{mRS}$ and depression, have been studied to some degree. As previously mentioned, Guzman et al. ${ }^{14}$ found that $\mathrm{mRS}$ scores improved postoperatively in adults and in children. In another study, Festa et al. ${ }^{9}$ measured depression with the Centers for Epidemiological Studies Depression scale. The authors found that $8(28 \%)$ of 29 patients showed evidence of depression. However, depression did not correlate with other neurocognitive findings, nor did it mirror the extent of cognitive impairment. In another study, only 2 (5.6\%) of 36 adults reported a moderate level of depression, defined as a Beck Depression Inventory-II score between 20 and $28 .{ }^{26}$ Depression prevalence has been found to be between $20 \%$ and $40 \%$ after acute neurological events such as stroke. ${ }^{37}$ Karzmark et al. ${ }^{26}$ rationalized the low prevalence of depression found 
in moyamoya patients by the progressive nature of the disease. They argued that moyamoya patients do not realize the severity of their condition in terms of its impact on their ability to perform daily activities. Self-reported depression may underestimate the true detriment to QOL; therefore, QOL assessment is a fertile ground for future studies.

Perfusion. Hypoperfusion has been hypothesized as a principal cause of the cognitive decline seen in adult moyamoya patients. ${ }^{9}$ In turn, improved perfusion postoperatively has been hypothesized to correlate with improved cognitive functioning. This relationship, though, has yet to be fully comprehended. To our knowledge only 1 series and 2 case reports have ever been published on the subject. All 3 studies revealed a correlation between cerebral perfusion as measured by perfusion-weighted MR imaging and neurological function pre- and postoperatively. ${ }^{3,24,51}$ Wityk et al. ${ }^{51}$ reported the improved cognitive function of a 24-year-old patient with moyamoya disease at a single follow-up evaluation 1 year postoperatively. The improved cognitive function was associated with a markedly increased perfusion of the involved hemisphere. Jefferson et al. ${ }^{24}$ reported serial neurocognitive testing of a 48-year-old moyamoya patient preoperatively and at 5 weeks postoperatively. They found a dramatic improvement in cognitive domains, specifically verbal learning and delayed recall, which correlated with a drastic increase in perfusion. Calviere et al. ${ }^{3}$ compared perfusion between 6 moyamoya patients with DCS and 4 moyamoya patients without DCS. Perfusion was significantly lower in those with DCS $(p=0.019)$ than in those without. Although these reports clearly revealed a correlation between increased perfusion and improved cognitive outcome, more data are needed and a prospective registry with serial cognitive and imaging follow-ups is warranted for further enhancement of our understanding of this observed phenomenon.

\section{Clinical Outcome}

Clinical outcome in adult moyamoya patients has been analyzed in terms of recurrent TIA, cerebral infarction, and hemorrhage. The postoperative risk of TIA and ischemic stroke decreases dramatically after revascularization. ${ }^{29,47}$ This decreased risk was quantified in a study of 43 post-EDAS adult moyamoya patients in North America. ${ }^{47}$ The primary outcomes studied were TIA, infarction, graft collateralization, mRS score, and perfusion changes. Postoperatively, $82 \%$ of the hemispheres showed increased perfusion, and $96 \%$ showed some collateral formation within the surgical region. The 5-year infarction-free survival rate was significantly increased, from $36 \%$ to $94 \%$ in hemispheres treated surgically ( $\mathrm{p}=$ $0.007)$. Surgical intervention reduced the risk of infarction by $89 \%$. Furthermore, the postoperative incidence of TIA was reduced from $58 \%$ on presentation to $7 \%$ during a median 41-month follow-up period (range 4-126 months). From these results, the authors concluded that indirect bypass procedures such as EDAS promote sufficient development of collateral vasculature, preventing future TIA and infarction.
Increased OEF can identify hemodynamic impairment, potentially predicting future ischemic events. ${ }^{39}$ An ongoing study by Zipfel et al. ${ }^{55}$ is prospectively analyzing the strength of OEF as a predictor of ipsilateral stroke and clinical outcome in moyamoya patients. These authors measure OEF levels with PET imaging on admission and at 1 and 3 years postoperatively. The interim results revealed that an elevation in cerebral OEF is frequently seen in moyamoya patients as a compensatory response to decreased perfusion. Hemodynamic impairment and increased OEF are predictors of stroke in carotid artery occlusion disorder, ${ }^{7}$ and Zipfel et al. are attempting to correlate these factors in moyamoya patients.

\section{Surgical Techniques and Outcomes}

In relation to the clinical and cognitive outcomes of both pediatric and adult patients, the efficacy of surgical procedures used to treat moyamoya disease has undergone much investigation. Although neither indirect nor direct bypass techniques have been proven superior to one another, surgical intervention is the only successful treatment for moyamoya disease..$^{19,20,29,32,50}$ One study found that the 5-year risk of ipsilateral stroke was reduced from $82 \%$ in conservatively treated patients to $17 \%$ in surgically treated patients. ${ }^{15}$ In a subsequent study, 25 pediatric moyamoya patients were followed, of whom 10 underwent surgical treatment, whereas 15 were treated conservatively. ${ }^{20}$ Surgical intervention was found to be more effective than conservative treatment in preventing poor cognitive outcome, especially if done early in the clinical course..$^{20,32}$

There is currently no clear consensus on the ideal timing for revascularization surgery.9,14,29,40 A national Japanese survey found $1.5 \%$ of patients with moyamoya disease to be asymptomatic; $21.2 \%$ of these patients became symptomatic within a mean of 3.7 years..$^{52}$ Narisawa et al..$^{40}$ followed up on adult moyamoya patients who underwent direct bypass in 63 hemispheres and were treated conservatively in 47 asymptomatic hemispheres. Six $(12.8 \%)$ of the 47 conservatively treated patients showed stenosis progression, whereas no cerebrovascular events were reported among surgically treated patients. The authors consequently drew the conclusion that asymptomatic moyamoya patients are at risk for disease progression and should undergo revascularization if they have ischemic symptoms or signs of stenotic progression.

The majority of the literature comparing direct and indirect bypass has found no significant difference in long-term outcome between the 2 procedures. However, in one study the 2 techniques were compared by retrospectively reviewing 368 moyamoya revascularization cases, and the investigators found that immediate postoperative ischemic events were more common after indirect revascularization (OR 5.8, 95\% CI 1.96-17.2). ${ }^{43}$ This could be due to the fact that indirect revascularization initiates a chronic sequence of chemical and biological events involving various growth factors that promote vessel formation only after 3-4 months postoperatively. ${ }^{31} \mathrm{On}$ the other hand, direct bypass immediately improves perfusion to the affected area. The literature suggests that a 
combination of direct and indirect bypass is preferable, and in the long term no significant difference in outcome exists between the 2 procedures. ${ }^{2,18,27}$

\section{Conclusions}

Moyamoya disease is an important cause of ischemic and hemorrhagic stroke. The combination of indirect and direct revascularization techniques is the recommended surgical approach and is beneficial to both clinical and cognitive outcomes. Neurocognitive impairment differs between adult and pediatric populations. Intelligence is the most affected cognitive parameter in children, whereas executive function is the most impaired parameter in adults. Very few studies have analyzed QOL in either population. Advancements in perfusion imaging will allow for a clearer understanding of the disease progression and long-term outcome of moyamoya patients.

\section{Disclosure}

The authors report no conflict of interest concerning the materials or methods used in this study or the findings specified in this paper.

Author contributions to the study and manuscript preparation include the following. Conception and design: Bendok, Rahme. Acquisition of data: Weinberg, Rahme. Analysis and interpretation of data: Bendok, Rahme. Drafting the article: Weinberg, Rahme, Aoun. Critically revising the article: Bendok, Batjer. Reviewed submitted version of manuscript: all authors. Approved the final version of the paper on behalf of all authors: Bendok.

\section{References}

1. Bowen M, Marks MP, Steinberg GK: Neuropsychological recovery from childhood moyamoya disease. Brain Dev 20:119-123, 1998

2. Burke GM, Burke AM, Sherma AK, Hurley MC, Batjer HH, Bendok BR: Moyamoya disease: a summary. Neurosurg Focus 26(4):E11, 2009

3. Calviere L, Catalaa I, Marlats F, Viguier A, Bonneville F, Cognard C, et al: Correlation between cognitive impairment and cerebral hemodynamic disturbances on perfusion magnetic resonance imaging in European adults with moyamoya disease. Clinical article. J Neurosurg 113:753-759, 2010

4. Chiu D, Shedden P, Bratina P, Grotta JC: Clinical features of moyamoya disease in the United States. Stroke 29:1347-1351, 1998

5. Darwish B, Besser M: Long term outcome in children with Moyamoya disease: experience with 16 patients. J Clin Neurosci 12:873-877, 2005

6. Delis DC, Kramer JH, Kaplan E, Holdnack J: Reliability and validity of the Delis-Kaplan Executive Function System: an update. J Int Neuropsychol Soc 10:301-303, 2004

7. Derdeyn CP, Grubb RL Jr, Powers WJ: Cerebral hemodynamic impairment: methods of measurement and association with stroke risk. Neurology 53:251-259, 1999

8. Ezura M, Yoshimoto T, Fujiwara S, Takahashi A, Shirane R, Mizoi K: Clinical and angiographic follow-up of childhoodonset moyamoya disease. Childs Nerv Syst 11:591-594, 1995

9. Festa JR, Schwarz LR, Pliskin N, Cullum CM, Lacritz L, Charbel FT, et al: Neurocognitive dysfunction in adult moyamoya disease. J Neurol 257:806-815, 2010

10. Filley CM: Clinical neurology and executive dysfunction. Semin Speech Lang 21:95-108, 2000

11. Fukui M: Current state of study on moyamoya disease in Japan. Surg Neurol 47:138-143, 1997
12. Gladsjo JA, Schuman CC, Evans JD, Peavy GM, Miller SW, Heaton RK: Norms for letter and category fluency: demographic corrections for age, education, and ethnicity. Assessment 6:147-178, 1999

13. Golby AJ, Marks MP, Thompson RC, Steinberg GK: Direct and combined revascularization in pediatric moyamoya disease. Neurosurgery 45:50-60, 1999

14. Guzman R, Lee M, Achrol A, Bell-Stephens T, Kelly M, Do $\mathrm{HM}$, et al: Clinical outcome after 450 revascularization procedures for moyamoya disease. Clinical article. J Neurosurg 111:927-935, 2009

15. Hallemeier CL, Rich KM, Grubb RL Jr, Chicoine MR, Moran CJ, Cross DT III, et al: Clinical features and outcome in North American adults with moyamoya phenomenon. Stroke 37:1490-1496, 2006

16. Hogan AM, Kirkham FJ, Isaacs EB, Wade AM, VarghaKhadem F: Intellectual decline in children with moyamoya and sickle cell anaemia. Dev Med Child Neurol 47:824-829, 2005

17. Houkin K, Yoshimoto T, Kuroda S, Ishikawa T, Takahashi A, Abe H: Angiographic analysis of moyamoya disease-how does moyamoya disease progress? Neurol Med Chir (Tokyo) 36:783-788, 1996

18. Ikezaki K: Rational approach to treatment of moyamoya disease in childhood. J Child Neurol 15:350-356, 2000

19. Imaizumi C, Imaizumi T, Osawa M, Fukuyama Y, Takeshita M: Serial intelligence test scores in pediatric moyamoya disease. Neuropediatrics 30:294-299, 1999

20. Imaizumi T, Hayashi K, Saito K, Osawa M, Fukuyama Y: Long-term outcomes of pediatric moyamoya disease monitored to adulthood. Pediatr Neurol 18:321-325, 1998

21. Ishii R, Takeuchi S, Ibayashi K, Tanaka R: Intelligence in children with moyamoya disease: evaluation after surgical treatments with special reference to changes in cerebral blood flow. Stroke 15:873-877, 1984

22. Ishikawa T, Houkin K, Kamiyama H, Abe H: Effects of surgical revascularization on outcome of patients with pediatric moyamoya disease. Stroke 28:1170-1173, 1997

23. Isobe M, Kuroda S, Kamiyama $\mathrm{H}$, Abe $\mathrm{H}$, Mitumori $\mathrm{K}$ : [Cerebral blood flow reactivity to hyperventilation in children with spontaneous occlusion of the circle of willis (moyamoya disease).] No Shinkei Geka 20:399-407, 1992 (Jpn)

24. Jefferson AL, Glosser G, Detre JA, Sinson G, Liebeskind DS: Neuropsychological and perfusion MR imaging correlates of revascularization in a case of moyamoya syndrome. AJNR Am J Neuroradiol 27:98-100, 2006

25. Karasawa J, Touho H, Ohnishi H, Miyamoto S, Kikuchi H: Long-term follow-up study after extracranial-intracranial bypass surgery for anterior circulation ischemia in childhood moyamoya disease. J Neurosurg 77:84-89, 1992

26. Karzmark P, Zeifert PD, Tan S, Dorfman LJ, Bell-Stephens TE, Steinberg GK: Effect of moyamoya disease on neuropsychological functioning in adults. Neurosurgery 62:10481052,2008

27. Kawaguchi S, Okuno S, Sakaki T: Effect of direct arterial bypass on the prevention of future stroke in patients with the hemorrhagic variety of moyamoya disease. J Neurosurg 93: 397-401, 2000

28. Kim CY, Wang KC, Kim SK, Chung YN, Kim HS, Cho BK: Encephaloduroarteriosynangiosis with bifrontal encephalogaleo(periosteal)synangiosis in the pediatric moyamoya disease: the surgical technique and its outcomes. Childs Nerv Syst 19: $316-324,2003$

29. Kuroda S, Houkin K: Moyamoya disease: current concepts and future perspectives. Lancet Neurol 7:1056-1066, 2008

30. Kuroda S, Houkin K, Ishikawa T, Nakayama N, Ikeda J, Ishii $\mathrm{N}$, et al: Determinants of intellectual outcome after surgical revascularization in pediatric moyamoya disease: a multivariate analysis. Childs Nerv Syst 20:302-308, 2004 


\section{Neurocognitive outcomes in patients with moyamoya disease}

31. Kuroda S, Houkin K, Ishikawa T, Nakayama N, Iwasaki Y: Novel bypass surgery for moyamoya disease using pericranial flap: its impacts on cerebral hemodynamics and long-term outcome. Neurosurgery 66:1093-1101, 2010

32. Kurokawa T, Tomita S, Ueda K, Narazaki O, Hanai T, Hasuo K, et al: Prognosis of occlusive disease of the circle of Willis (moyamoya disease) in children. Pediatr Neurol 1:274-277, 1985

33. Matsushima T, Inoue TK, Suzuki SO, Inoue T, Ikezaki K, Fukui M, et al: Surgical techniques and the results of a frontotemporo-parietal combined indirect bypass procedure for children with moyamoya disease: a comparison with the results of encephalo-duro-arterio-synangiosis alone. Clin Neurol Neurosurg 99 (Suppl 2):S123-S127, 1997

34. Matsushima Y, Aoyagi M, Koumo Y, Takasato Y, Yamaguchi T, Masaoka H, et al: Effects of encephalo-duro-arterio-synangiosis on childhood moyamoya patients - swift disappearance of ischemic attacks and maintenance of mental capacity. Neurol Med Chir (Tokyo) 31:708-714, 1991

35. Matsushima Y, Aoyagi M, Masaoka H, Suzuki R, Ohno K: Mental outcome following encephaloduroarteriosynangiosis in children with moyamoya disease with the onset earlier than 5 years of age. Childs Nerv Syst 6:440-443, 1990

36. Matsushima Y, Aoyagi M, Nariai T, Takada Y, Hirakawa K: Long-term intelligence outcome of post-encephalo-duro-arterio-synangiosis childhood moyamoya patients. Clin Neurol Neurosurg 99 (Suppl 2):S147-S150, 1997

37. McDermott S, Moran R, Platt T, Issac T, Wood H, Dasari S: Depression in adults with disabilities, in primary care. Disabil Rehabil 27:117-123, 2005

38. Miyamoto S, Akiyama Y, Nagata I, Karasawa J, Nozaki K, Hashimoto N, et al: Long-term outcome after STA-MCA anastomosis for moyamoya disease. Neurosurg Focus 5(5):e5, 1998

39. Morimoto M, Iwama T, Hashimoto N, Kojima A, Hayashida $\mathrm{K}$ : Efficacy of direct revascularization in adult Moyamoya disease: haemodynamic evaluation by positron emission tomography. Acta Neurochir (Wien) 141:377-384, 1999

40. Narisawa A, Fujimura M, Tominaga T: Efficacy of the revascularization surgery for adult-onset moyamoya disease with the progression of cerebrovascular lesions. Clin Neurol Neurosurg 111:123-126, 2009

41. Ohtaki M, Uede T, Morimoto S, Nonaka T, Tanabe S, Hashi K: Intellectual functions and regional cerebral haemodynamics after extensive omental transplantation spread over both frontal lobes in childhood moyamoya disease. Acta Neurochir (Wien) 140:1043-1053, 1998

42. Patel MD, McKevitt C, Lawrence E, Rudd AG, Wolfe CD: Clinical determinants of long-term quality of life after stroke. Age Ageing 36:316-322, 2007

43. Sakamoto T, Kawaguchi M, Kurehara K, Kitaguchi K, Furuya $\mathrm{H}$, Karasawa J: Risk factors for neurologic deterioration after revascularization surgery in patients with moyamoya disease. Anesth Analg 85:1060-1065, 1997
44. Sato H, Sato N, Tamaki N, Matsumoto S: Chronic low-perfusion state in children with moyamoya disease following revascularization. Childs Nerv Syst 6:166-171, 1990

45. Scott RM, Smith JL, Robertson RL, Madsen JR, Soriano SG, Rockoff MA: Long-term outcome in children with moyamoya syndrome after cranial revascularization by pial synangiosis. J Neurosurg 100 (2 Suppl Pediatrics):142-149, 2004

46. So Y, Lee HY, Kim SK, Lee JS, Wang KC, Cho BK, et al: Prediction of the clinical outcome of pediatric moyamoya disease with postoperative basal/acetazolamide stress brain perfusion SPECT after revascularization surgery. Stroke 36: 1485-1489, 2005

47. Starke RM, Komotar RJ, Hickman ZL, Paz YE, Pugliese AG, Otten ML, et al: Clinical features, surgical treatment, and long-term outcome in adult patients with moyamoya disease. Clinical article. J Neurosurg 111:936-942, 2009

48. Suzuki J, Takaku A: Cerebrovascular "moyamoya" disease. Disease showing abnormal net-like vessels in base of brain. Arch Neurol 20:288-299, 1969

49. Uchino K, Johnston SC, Becker KJ, Tirschwell DL: Moyamoya disease in Washington State and California. Neurology 65: 956-958, 2005

50. Veeravagu A, Guzman R, Patil CG, Hou LC, Lee M, Steinberg GK: Moyamoya disease in pediatric patients: outcomes of neurosurgical interventions. Neurosurg Focus 24(2):E16, 2008

51. Wityk RJ, Hillis A, Beauchamp N, Barker PB, Rigamonti D: Perfusion-weighted magnetic resonance imaging in adult moyamoya syndrome: characteristic patterns and change after surgical intervention: case report. Neurosurgery 51:14991506,2002

52. Yamada M, Fujii K, Fukui M: [Clinical features and outcomes in patients with asymptomatic moyamoya disease-from the results of nation-wide questionnaire survey.] No Shinkei Geka 33:337-342, 2005 (Jpn)

53. Yun TJ, Cheon JE, Na DG, Kim WS, Kim IO, Chang KH, et al: Childhood moyamoya disease: quantitative evaluation of perfusion MR imaging - correlation with clinical outcome after revascularization surgery. Radiology 251:216-223, 2009

54. Zakzanis KK, Mraz R, Graham SJ: An fMRI study of the Trail Making Test. Neuropsychologia 43:1878-1886, 2005

55. Zipfel GJ, Sagar J, Miller JP, Videen TO, Grubb RL Jr, Dacey RG Jr, et al: Cerebral hemodynamics as a predictor of stroke in adult patients with moyamoya disease: a prospective observational study. Neurosurg Focus 26(4):E6, 2009

Manuscript submitted February 16, 2011.

Accepted March 22, 2011.

Address correspondence to: Bernard R. Bendok, M.D., Departments of Neurological Surgery and Radiology, Northwestern University Feinberg School of Medicine, 676 North St. Clair Street, Suite 2210, Chicago, Illinois 60611. email: bbendok@nmff.org. 